Classification

Physics Abstracts

$72.10-71.27-72.15$

\title{
Impurity scattering in 1D ring of interacting electrons
}

\author{
Georges Bouzerar $\left(^{*}\right)$ and Didier Poilblanc $\left({ }^{* *}\right)$ \\ Groupe de Physıque Théorique, Laboratoire de Physique Quantique, Université Paul Sabatier, \\ 31062 Toulouse, France
}

(Received 18 May 1994, accepted 28 July 1994)

\begin{abstract}
The stability of the 1D Luttinger liquid phase of interacting spinless particles $(t-V$ model $)$ to an on-site diagonal disorder is studied by exact diagonalization of small 1D rings up to 22 sites. We discuss our numerical results in the light of the phase diagram predicted by the Renormalization Group analysis. In a finite range of attractive interactions (around $V / t \sim-0.5$ ) the metallic phase (with superconducting fluctuations) seems to be stable up to some small value of the disorder parameter. On the other hand, in the repulsive case, the Impurity potential clearly leads to a strong localization of the particles.
\end{abstract}

The aim of this paper is to study the interplay between interactions and disorder in a $1 D$ Luttinger liquid. For lattice models of spinless fermions with short range repulsive interactions on-site diagonal disorder is believed to produce localization of the particles. However, for an attractive interaction, superconducting critical fluctuations appear and the influence of the disorder is not so clear.

To be more specific we consider the following Hamiltonian describing a $N$-site chain of spinless fermions in the presence of disorder:

$$
\mathcal{H}=-t / 2 \sum_{\imath}\left(\exp (2 i \pi \Phi / N) c_{\imath}^{\dagger} c_{\imath+1}+\text { h.c. }\right)+V \sum_{\imath} n_{\imath} n_{\imath+1}+\sum_{\imath} w_{\imath} n_{\imath}
$$

where $w_{2}$ are on-site energies chosen randomly between $-W / 2$ and $W / 2, V$ is the nearest neighbour interaction and $\Phi$ is a magnetic flux through the ring (measured in units of flux quantum $\Phi_{0}=h / e$ ). So far we set $t=1$. The magnetic flux will be useful later on to test the sensitivity of the system to a change in the boundary conditions.

In the absence of disorder ( $W=0$ ) the model is integrable. At half-filling, for repulsive interaction $(V>0)$, Umklapp scattering dominates and a metal-insulator transition occurs at $V=1$ with a charge gap opening up for $V>1$. Away from commensurability (i.e. away

${ }^{*}$ ) E-mail: georges@siberı.ups-tlse.fr.

$\left({ }^{* *}\right)$ E-mall: didier@occitana.ups-tlse.fr. 
from half-filling), the system remains metallic even for $V>1$. For $V<-1$ (and arbitrary filling) the metallic phase becomes unstable with respect to phase separation. The metallic phase belongs to the class of the Luttinger liquid and the critical exponents of the correlation functions have been calculated in detail by Haldane [1]. Charge density wave (superconducting) fluctuations dominate in the repulsive (attractive) case. The critical exponents depend on a single parameter $K_{\rho}$. At half filling $K_{\rho}$ is given by a simple analytic form, $K_{\rho}=\frac{1}{2(1-\mu / \pi)}$ where, using Haldane's notation, $\mu$ is related to the electron interaction by $\cos \mu=V$. On the other hand, the velocity is given by $u_{\rho}=\pi(\sin \mu / \mu)$. Note that, by a standard WignerJordan transformation, Hamiltonian (1) can be mapped onto the anisotropic XXZ spin $1 / 2$ model where the $V$ term ( $t$ term) corresponds to the diagonal (spin flip) coupling. The on-site disorder takes the form of a coupling of the localized spins with random magnetic fields.

Weak disorder can often be treated perturbatively and resummation of certain classes of diagrams can be performed using a renormalization group (RG) analysis. To lowest order the $\mathrm{RG}$ equations [2,3] for the Luttinger parameters of the disordered spinless fermions system can be written as (neglecting Umklapp processes),

$$
\begin{gathered}
\frac{\mathrm{d} K_{\rho}}{\mathrm{d} l}=-\left(K_{\rho}^{2}\right) \frac{D_{\xi}}{2} \\
\frac{\mathrm{d} D_{\xi}}{\mathrm{d} l}=\left(3-2 K_{\rho}\right) D_{\xi} \\
\frac{\mathrm{d} u_{\rho}}{\mathrm{d} l}=-(1 / 2) u_{\rho} K_{\rho} D_{\xi}
\end{gathered}
$$

where $D_{\xi}=\frac{W^{2} N}{6 \pi u_{\rho}^{2}}$ is a dimensionless disorder parameter, and $l=\ln (N)$. There is a critical value for $K_{\rho}, K_{\rho}^{\mathrm{c}}=3 / 2$ obtained for $V=V_{\mathrm{c}}=-1 / 2$. Close to the critical point $K_{\rho}^{\mathrm{c}}$ this set of equations become 1dentical to the Kosterlitz-Thouless (KT) RG equations describing the transition in a $2 \mathrm{D}$ Coulomb gas between a phase of dipoles and a plasma phase where the pairs start to unbind (disordered phase). In our case the role played by the disorder corresponds in the KT picture to the temperature. The delocalized phase can be interpreted as a phase of electron pairs, moving on the lattice. For vanishing disorder and $K_{\rho}<3 / 2,(V>-1 / 2)$, equation (3) indicates that disorder renormalizes to $\infty$, the fixed point being a localized phase while for $K_{\rho}>3 / 2$ (1.e. $-1<V<-1 / 2$ ) disorder renormalizes to 0 and the system is delocalized. Hence for $K_{\rho}>3 / 2$ we expect a transition from a delocalized to a localized phase at some value $W(V)=W_{\mathrm{c}}$.

The critical line at small but finite $W$ can be obtained by eliminating $l$ between equations (2) and (3). Using $D_{\xi}^{\mathrm{c}}=\frac{W_{\mathrm{c}}^{2}}{6 \pi u_{\rho}^{2}}$, we can easily deduce $W_{\mathrm{c}}$ versus $V$. We chose to start the renormalization process with a single site $(l=0)$. Figure 1 corresponds to $W_{\mathrm{c}}$ versus $V$ and is in qualitative agreement with the phase diagram of reference [5]. We get a maximum value of the disorder of $W_{\max } \propto 2.3$. Note that the multiplicative prefactor $\frac{1}{6 \pi u_{\rho}^{2}} \sim 0.015$ in the dimensionless parameter $D_{\xi}$ is very small so that even $W_{\max }$ correspond to a small $D_{\xi}^{\max } \propto 0.08$.

As reported in our previous work on the calculation of the persistent currents in $1 \mathrm{D}$ interacting disordered systems [4], the charge stiffness $D$,

$$
D=\frac{N}{4 \pi^{2}}\left(\frac{\partial^{2} E(\Phi)}{\partial \Phi^{2}}\right)_{\Phi=\Phi_{\mathrm{m}}} .
$$




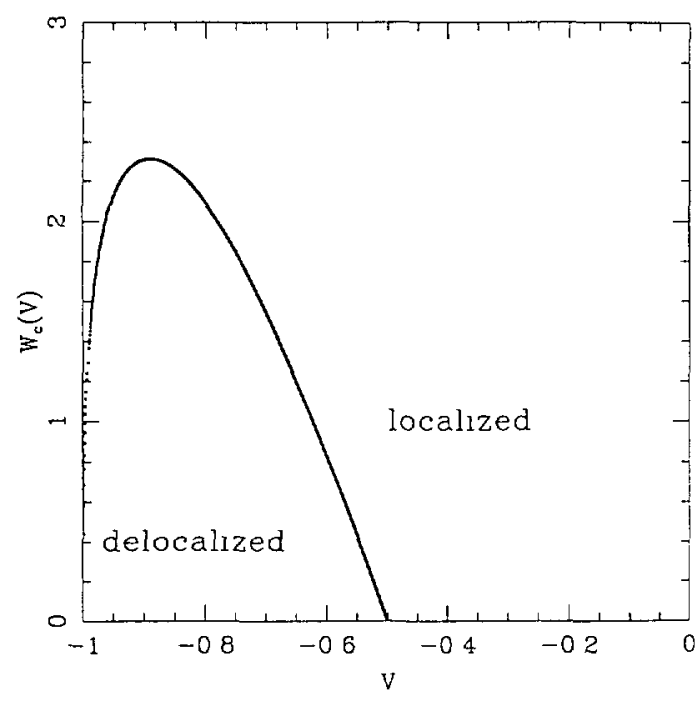

Fig. 1

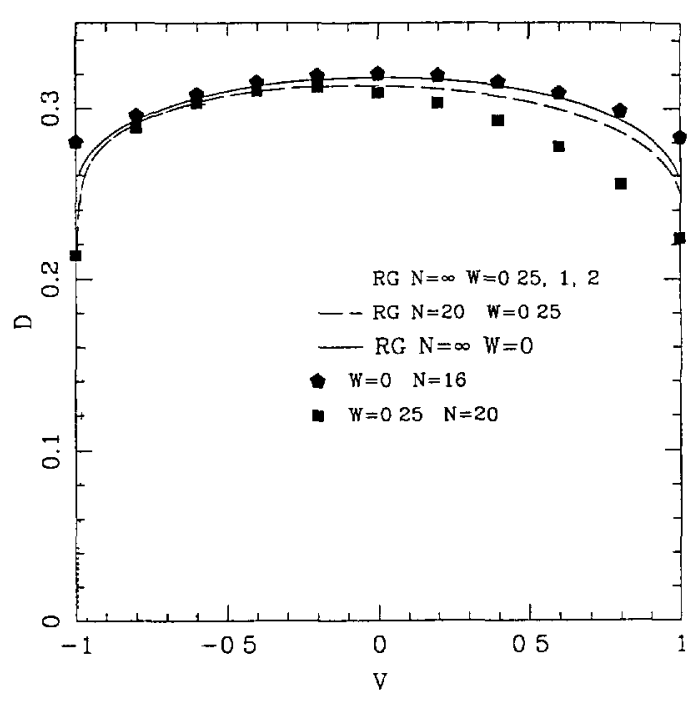

Fig. 2

Fig. 1. - Phase diagram representing the critical line $W_{c}(V)$.

Fig. 2. - Drude weight calculated for $-1<V<1$ by (i) the RG equations for $N=16,20, \infty$ and $W=0,0.25,1$ and 2 (lines). (ii) exact diagonalizations for $N=16, W=0$ and $N=20, W=0.25$ (dots). We averaged over at least 100 realisations of the disorder in the case $W=0.25$.

is a relevant parameter to characterize the nature of the different phases. $\Phi_{m}=0$ or $1 / 2$ is the location of the minimum of $E(\Phi)$. In the localized phase and in the thermodynamic limit $D=0$, while a delocalized phase can be characterızed by $D_{\infty} \neq 0$. Such a criterion was also used to investigate the Mott transition in pure interacting rings [6]. The expression of the Drude weight $\pi D$ in term of the Luttinger liquid parameters is,

$$
\pi D=\frac{u_{\rho} K_{\rho}}{2}
$$

Using the RG equations we calculate $D_{\infty}$ for $V$ varying from -1 to 1 and begining the procedure with $N=1$. As a reference the Drude weight at $W=0$ calculated by using Haldane's analitycal expressions is shown in figure 2. In the presence of disorder $(W=0.25,1$, and 2$)$ iterating equations (2) and (3) to $N=\infty$ gives $D_{\infty}$ displayed in figure 2 as a function of $V$. We observe the decay of the Drude weight for increasing $W$. When $W$ is larger than the critical value $W_{\max }, D_{\infty}=0$ for any $V$, the system being in the localized phase.

We now compare the RG results with the data obtained by exact diagonalizations. We extend here a recent work [7] to larger system sizes. First, we briefly describe the method used to calculate numerically the Drude weight. Using the Lanczos algorithm we diagonalize exactly the Hamiltonian (1), and calculate the groundstate energy vs. $\Phi$. We can then deduce the Drude weight $\pi D$, from the expression of the charge stiffness given by (5). Note that $\Phi_{m}$ depends on the parity of the number of electrons. We have performed the calculations for $N=12,14,16,20$ and 22 at half filling for the pure case and for $W=0.25$ and $W=1, V$ varying from -1 to 1 . An average over at least 100 realisations of the disorder was performed (in general 300 except for $N=22$ ). A good agreement between exact calculations and analytical 


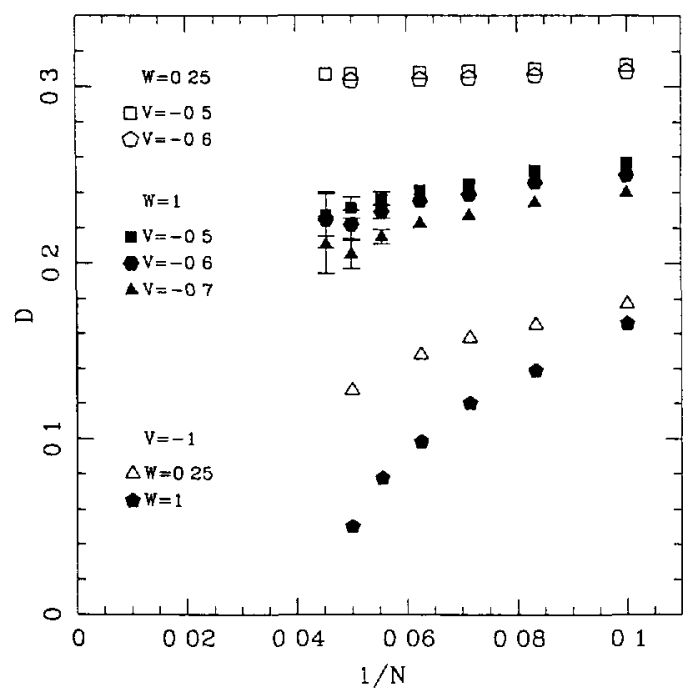

Fig. 3. $-D$ versus $1 / N$ for $W=0.25$ and 1 and $V=-1,-0.7,-0.6,-0.5$. When not displayed, error bars are smaller than the size of the dots. The dots for $W=0.25, V=-1$ have been shifted down by 0.9 .

results is found for $W=0$ and $V$ not too close to the limiting values $V=1,-1$ (Fig. 2).

As a test we compare (Fig. 2) the data obtained for weak disorder $W=0.25$ by (i) using the RG equations up to a size $N=20$ and (ii) numerical diagonalization. We observe that, for $V<-1 / 2, D$ is weakly affected by the disorder. For sufficently attractive interaction, say $-1<V<-1 / 2$, the data seem to converge rapidly to $D_{\infty}$.

Figure 3 represents the scaling of $D$ vs. $1 / N$ for fixed $W=0.25$ and $W=1$ and the parameter $V=-0.5,-0.6$ and -1 . We observe that $D$ seems to scale to a finite value in both cases $V=-0.5$ and -0.6 . The critical case $V=-0.5$ deserves special attention. Indeed, the RG predicts that $D_{\infty}$ is finite only for $-1<V<-0.5$, and zero elsewhere. However we do not observe any qualitative difference between $V=-0.5$ and -0.6 . At this point we can only say that, either (i) the system is in a delocalized phase or (ii) the localization length $\xi>>1$ as predicted by the RG analysis. However, the results are qualitatively different for larger values of $W$, say $W=1$; in contrast to the $R G$ results the phase is always localized even for $-1<V<-0.5$.

To conclude, our results are in qualitative agreement with [7]. They are consistent with the existence of a delocalized phase in a restricted region of the parameter space. However, in contrast to RG, the region of stability of the disordered phase seems to be centered around $V=-0.5$. We can not exclude that the localization length $\xi>>1$. In any case, the size of the stability region must be significantly smaller than the $\mathrm{RG}$ prediction ${ }_{1}$ e. $W_{\max }<W_{\max }^{\mathrm{RG}}$.

\section{Acknowledgments.}

We thank the Institut du Developpement et des Ressources en Informatique Scientifique (IDRIS), Orsay, (France) for allocation of CPU time on the Cray C98 and T. Giamarchi for useful discussions. D.P. also acknowledges support from the EEC Human Capital and Mobil1ty Program under grant CHRX-CT93-0332. Laboratoire de Physique Quantique (Toulouse) is Unité Associée No. URA505 du CNRS. 


\section{References}

[1] Haldane F.D.M., Phys. Rev. Lett. 45 (1980) 1358.

[2] Apel W., J. Phys. C 15 (1982) 1973.

[3] Giamarchi T., PhD Thesis (1987).

[4] Bouzerar G., Poilblanc D. and Montambaux G., Phys. Rev. B 49 (1994) 8258.

[5] Doty A. and Fisher D.S., Phys. Rev. B 45 (1992) 2167.

[6] Scalapino D.J. et al., Phys. Rev. B 44 (1993) 6909.

[7] Runge K.J. and Zymanyı G.T., Phys. Rev. B 49 (1994) 15212. 